\title{
Constant-free, randomized a posteriori error estimators for parameter-dependent partial differential equations
}

\author{
Kathrin Smetana* Olivier Zahm ${ }^{* *}$ Anthony T. Patera ${ }^{* * *}$ \\ * University of Twente, 7500 AE Enschede, The Netherlands (e-mail: \\ k.smetana@utwente.nl). \\ ** Massachusetts Institute of Technology, Cambridge, MA 02139-4307 \\ USA, (e-mail: zahmo@mit.edu) \\ *** Massachusetts Institute of Technology, Cambridge, MA 02139-4307 \\ USA, (e-mail: patera@mit.edu)
}

Keywords: A posteriori error estimation, reduced basis method, parametrized partial differential equations, randomization, concentration inequalities, high dimensional probability, adjoint method

\section{INTRODUCTION}

During the last decades (numerical) simulations based on partial differential equations (PDEs) have considerably gained importance in engineering applications, life sciences, environmental issues, and finance. However, especially when multiple simulation requests or a real-time simulation response are desired, standard methods such as finite elements (FE) are prohibitive. Model reduction approaches such as the reduced basis (RB) method, which we will consider here, have been developed to tackle such situations (see for instance Haasdonk (2017); Quarteroni et al. (2016); Hesthaven et al. (2016) for an overview). The key concept of the RB method is to prepare a problemadapted low-dimensional subspace of the high-dimensional (FE) discretization space in a possibly expensive offline stage to realize a fast simulation response by Galerkin projection on that low-dimensional space in the subsequent online stage.

To assess the approximation error caused by the RB method in the online stage a reliable and efficient a posteriori error estimator has been derived in Veroy et al. (2003). However, for inf-sup stable problems such as acoustics problems the estimation of the inf-sup constant still poses a challenge and the existing methods often result in rather pessimistic results and thus pessimistic error bounds. We propose a constant-free, probabilistic a posteriori error estimator that does not require to estimate any stability constants and is both reliable and efficient at (given) high probability. Here, we extend the approach in Cao and Petzold (2004); Homescu et al. (2005), where the solution of an adjoint problem with random conditions at the final time is employed to estimate the approximation error for ordinary differential equations.

\section{THE REDUCED BASIS METHOD FOR INF-SUP STABLE PARAMETRIZED PDES}

\subsection{Problem setting}

Let $D \subset \mathbb{R}^{d}, d=1,2,3$ be a bounded Lipschitz domain, $\mathcal{P}$ denote the set of admissible parameters, and introduce a Hilbert space $H_{0}^{1}(D) \subset X \subset H^{1}(D)$. Moreover, we introduce a linear operator $A(\mu): X \rightarrow X^{\prime}$ that is infsup stable and bounded, i.e.

$$
\begin{aligned}
0<\beta \leq \beta(\mu) & :=\inf _{v \in X} \sup _{w \in X} \frac{\langle A(\mu) v, w\rangle}{\|v\|_{X}\|w\|_{X}} \text { and } \\
\gamma(\mu) & :=\sup _{v \in X} \sup _{w \in X} \frac{\langle A(\mu) v, w\rangle}{\|v\|_{X}\|w\|_{X}} \leq \gamma<\infty,
\end{aligned}
$$

where $X^{\prime}$ denotes the dual space of $X$ and $\langle\cdot, \cdot\rangle$ the duality pairing.

We consider the following parameter-dependent PDE: For any given $\mu \in \mathcal{P}$ find $u(\mu) \in X$ such that

$$
A(\mu) u(\mu)=f(\mu) \text { in } X^{\prime},
$$

where $f(\mu) \in X^{\prime}$ is a given continuous linear form.

\subsection{The high-dimensional discretization}

Next, we introduce a conforming high-dimensional (FE) space $X^{\mathcal{N}} \subset X$ of dimension $\mathcal{N}$ and a so-called truth solution $u^{\mathcal{N}}(\mu)$ that is defined as the solution of

$$
A(\mu) u^{\mathcal{N}}(\mu)=f(\mu) \text { in } X^{\mathcal{N} \prime} \text {. }
$$

Note that in order to simplify the presentation we do not introduce suitable discrete linear operators as we believe the respective definition to be clear from the actual setting.

\subsection{The reduced basis method in a nutshell}

We assume that we have constructed an RB space $X^{N}:=$ $\operatorname{span}\left\{\phi_{1}, \ldots, \phi_{N}\right\}$ in the offline stage say via a greedy algorithm as introduced in Veroy et al. (2003), relying on 
the high-dimensional discretization. We may then define an RB approximation $u^{N}(\mu) \in X^{N}$ as the solution of

$$
A(\mu) u^{N}(\mu)=f(\mu) \text { in } X^{N \prime} \text {. }
$$

Note that thanks to a suitable offline/online-decomposition the computation of $u^{N}(\mu)$ in the online stage does not scale in the dimension $\mathcal{N}$ of the high-dimensional discretization (see for instance Haasdonk (2017); Quarteroni et al. (2016); Hesthaven et al. (2016) for details).

To assess the approximation error $\left\|u^{\mathcal{N}}(\mu)-u^{N}(\mu)\right\|_{X}$ one may introduce the residual

$$
r(\mu):=f(\mu)-A(\mu) u^{N}(\mu) \in X^{\mathcal{N} \prime} .
$$

It is then straightforward to show that we have

$$
\left\|u^{\mathcal{N}}(\mu)-u^{N}(\mu)\right\|_{X} \leq \frac{1}{\beta(\mu)}\|r(\mu)\|_{X^{\mathcal{N}}} .
$$

While the dual norm of the residual can be efficiently computed via the Riesz representation, estimation of $\beta(\mu)$ remains a challenge. The Successive Constraint Method introduced in Huynh et al. (2007) yields a lower bound for $\beta(\mu)$ which may however be rather pessimistic.

\section{A RANDOMIZED A POSTERIORI ERROR ESTIMATOR}

To derive a randomized a posteriori error estimator we rely on results similar to the restricted isometry property employed in compressed sensing. In detail, we introduce a matrix $B \in \mathbb{R}^{K \times \mathcal{N}}$ whose entries are mutually independent standard Gaussian random variables. Then, for a vector $\underline{x} \in \mathbb{R}^{\mathcal{N}}$ we have that for a given $\varepsilon \in \mathbb{R}, \varepsilon<1$ the result

$$
(1-\varepsilon)\|\underline{x}\|_{2}^{2} \leq\|\underline{B x}\|_{2}^{2} \leq(1+\varepsilon)\|\underline{x}\|_{2}^{2}
$$

holds true at a (given) probability of at most $1-\delta$ if $K \geq \bar{K}(\delta, \varepsilon)$ (see for instance Vershynin (2012)). Here, $\|\cdot\|_{2}$ denotes the Euclidean norm. Note that in contrast to our approach the authors of Cao and Petzold (2004); Homescu et al. (2005) employ the small sample statistical method as proposed in Kenney and Laub (1994) that relies on random vectors which are uniformly distributed on the sphere $S^{\mathcal{N}-1}$.

Inspired by the results in Cao and Petzold (2004); Homescu et al. (2005) we introduce dual problems

$$
\underline{A}^{*}(\mu) \underline{\psi}_{i}(\mu)=\underline{B}_{(i,:)}, \quad i=1, \ldots, K,
$$

where $\underline{A}^{*}(\mu)$ denotes the stiffness matrix associated with the adjoint operator $A^{*}(\mu)$ of $A(\mu)$ and $\underline{B}_{(i,:)}$ denotes the $i$-th row of the matrix $\underline{B}$. We may then use the dual solutions $\underline{\psi}_{i}(\mu)$ to define a probabilistic a posteriori error estimator that is a reliable and efficient bound of the error $\left\|u^{\mathcal{N}}(\mu)-u^{N}(\mu)\right\|_{X}$ at given probability $1-\delta$. However, as solving (9) for one $i$ is as expensive as solving the primal truth problem (4) this estimator is not computationally feasible in the online stage.

In order to obtain an a posteriori error estimator that can be computed in the online stage in a complexity that does not depend on the dimension of the high-dimensional space $X^{\mathcal{N}}$, we also introduce an RB approximation of the dual problems (9). Here, we propose and compare different computational strategies for the generation of the dual RB space. If the RB approximation errors of the dual problems are small, the error $\left\|u^{\mathcal{N}}-u^{N}(\mu)\right\|_{X}$ can be bounded, with high probability, from below and above by this onlineefficient a posteriori error estimator times a given constant. For more details see Smetana et al. (2018).

\section{REFERENCES}

Cao, Y. and Petzold, L. (2004). A posteriori error estimation and global error control for ordinary differential equations by the adjoint method. SIAM J. Sci. Comput., 26(2), 359-374.

Haasdonk, B. (2017). Reduced Basis Methods for Parametrized PDEs - A Tutorial Introduction for Stationary and Instationary Problems, in: Model Reduction and Approximation: Theory and Algorithms, Editors: P. Benner, A. Cohen, M. Ohlberger, and K. Willcox. SIAM Publications, Philadelphia, PA.

Hesthaven, J.S., Rozza, G., and Stamm, B. (2016). Certified Reduced Basis Methods for Parametrized Partial Differential Equations. SpringerBriefs in Mathematics. Springer International Publishing.

Homescu, C., Petzold, L.R., and Serban, R. (2005). Error estimation for reduced-order models of dynamical systems. SIAM J. Numer. Anal., 43(4), 1693-1714.

Huynh, D.B.P., Rozza, G., Sen, S., and Patera, A.T. (2007). A successive constraint linear optimization method for lower bounds of parametric coercivity and inf-sup stability constants. C. R. Math. Acad. Sci. Paris, 345(8), 473-478.

Kenney, C.S. and Laub, A.J. (1994). Small-sample statistical condition estimates for general matrix functions. SIAM J. Sci. Comput., 15(1), 36-61.

Quarteroni, A., Manzoni, A., and Negri, F. (2016). Reduced Basis Methods for Partial Differential Equations. La Matematica per il 3+2. Springer International Publishing.

Smetana, K., Zahm, O., and Patera, A.T. (2018). Constant-free, randomized residual-based error estimators for parameter-dependent equations. Unpublished.

Veroy, K., Prud'homme, C., Rovas, D.V., and Patera, A.T. (2003). A posteriori error bounds for reduced-basis approximation of parametrized noncoercive and nonlinear elliptic partial differential equations. In Proceedings of the 16th AIAA Computational Fluid Dynamics Conference, volume 3847 .

Vershynin, R. (2012). Introduction to the non-asymptotic analysis of random matrices. In Compressed sensing, 210-268. Cambridge Univ. Press, Cambridge. 